\title{
Optimal "Sporadic" and Systematic Preventive Maintenance Policy for Leased Equipment under Various Operating Conditions
}

\author{
Jérémie Schutz \\ Université de Lorraine, LGIPM, EA 3096, Metz, F-57045, France
}

\begin{abstract}
In this paper, the considered system corresponds to a specific equipment proposed for leasing. This equipment is leased to several lessees who use it under various working conditions. The aims of this paper consist in determining optimal maintenance plans which minimize maintenance costs. Two maintenance policies are compared : systematic policy (imperfect preventive maintenance actions are performed, with the same effectiveness factor, after each mission) and "sporadic" policy (imperfect preventive maintenance action can be performed after a mission with its optimal effectiveness factor).
\end{abstract}

Keywords: Leased equipment, Working conditions, Maintenance policy, Finite horizon.

\section{Introduction}

In this research area, the first work on the themes of maintenance is usually attributed to Barlow and Hunter [1]. In this work, these authors were interested in determining an optimal time for replacement equipment thanks to the development of two major strategies known under the name "Age-Based Maintenance" and "Block-Based Maintenance". Subsequently, many authors have determined, for these periods of renewal, maintenance policies in order to minimize maintenance costs. Also, we can refer to the work of Nakagawa [7] who has made a very significant contribution in this area with periodical and sequential policies. Periodic policies are characterized by preventive maintenance actions performed at constant time intervals. Sequential policies consist in determining the optimal interval between each preventive action. Generally, for these policies, preventive maintenance actions are considered perfect (the equipment is restored to a state "As Good As New"). Corrective actions are considered minimal (the failure rate of equipment remains unchanged "As Bad As Old"). In 1988, Kijima et al. [5] proposed a first model for imperfect maintenance. It is characterized by an age reduction of the system. This reduction corresponds to a proportional amount of the time elapsed since the previous preventive maintenance activity. Nakagawa [8] proposed an alternative approach to modeling imperfect maintenance. It is characterized by an intensity increase in the failure rate after every

B. Grabot et al. (Eds.): APMS 2014, Part I, IFIP AICT 438, pp. 451458 2014.

(C) IFIP International Federation for Information Processing 2014 
preventive maintenance. However, after each imperfect action, the failure rate is reduced to zero ; the system can be considered new. All these models assume that the working of the studied system is constant over time. Alas, in reality, an equipment can operate under different operational conditions (e.g. production rate) or environmental conditions (e.g. temperature). Therefore, the degradation of the system depends on these conditions and the equipment can thus degrade faster or more slowly. In the literature, two models are used to represent the degradation variations. Accelerated Life Model (ALM) and Proportional Hazards Model (PHM) influence the reliability function (or hazard function) by adding a risk function [6] [3]. The ALM changes the age of the system while the PHM defines a variation of the failure rate proportional to the working conditions.

Thanks to over half a century of research work and considering many important economic crises in recent years, recent work has sought to develop and implement maintenance policies for leased equipment. In recent work, the most notable contributions are those of Jaturonnatee et al. 4, Pongpech and Murthy [9] and Yeh et al. ([11, [12, [13], 14]). In these works, we find the usual policies such as sequential and periodic ones with imperfect maintenance based on the age reduction or failure rate increase. In 2007, Yeh and Chang offered an innovative policy where preventive maintenance actions are realized when the failure rate reaches a preset threshold.

Based on these observations, the aims of this paper consist in determining optimal maintenance plans which minimize maintenance costs for a leased equipment. This equipment is leased to several lessees with various working conditions. The remainder of this paper is organized as follows. Section 2 gives a studied system description, notations and working assumptions. In section 3, mathematical formulations of the maintenance policies and resolution methods are proposed. A numerical example is presented in the section 4. Finally, section 5 gives the conclusion and the future work.

\section{Problem Description}

In this paper, the considered system corresponds to a specific equipment proposed for leasing. Lessees are placed in a queue and obtain equipment for the desired period (duration of the mission) as it becomes available. Between two missions, this equipment can undergo imperfect preventive maintenance actions (based on age reduction) performed by the lessor (owner). These actions do not have negligible durations unlike the minimal corrective maintenance activities. The duration to perform preventive actions is a time percentage $\alpha$ of the length of all missions. The minimal corrective maintenance actions are performed during the missions by the lessor, when the system fails. The effect of working conditions, for a mission $m$, is based on the proportional hazard model (PHM) where they are modeled by a risk function $g_{m}$ [2]. So, the hazard function is given by $g_{m} \cdot \lambda(t)$. 


\section{$2.1 \quad$ Notations}

Throughout the paper, the following notations will be used:

- $\mathcal{M}$ : Missions Vector to perform

- $\delta_{m}$ : Duration of the mission $m$

$-\lambda(t)$ : Hazard function for nominal conditions

$-\Gamma(\cdot)$ : Total cost of maintenance policy

$-\Phi(\cdot)$ : Average number of failures

- $\rho$ : Effectiveness factor of preventive maintenance action

- $C_{C M}$ : Corrective maintenance action cost

- $C_{P M}(\rho)$ : Preventive maintenance action cost based on effectiveness factor $\rho$

Other notations used in this document are defined below equations.

\section{Mathematical Formulation}

\subsection{Minimal Maintenance Policy}

The purpose of this policy consists only in maintaining the system in a state of working to assess the contribution of preventive maintenance policies. This maintenance policy consists solely of minimal corrective maintenance actions. These activities are carried out to overcome the immobilizing failures that arise over time. Maintenance total cost is given by:

$$
\Gamma(\mathcal{M})=C_{C M} \cdot \phi(\mathcal{M})
$$

Usually, when the system operates under constant working conditions during a period $[0, T]$, the average number of failures corresponds to the cumulative hazard function at the time $T$. However, in this research, the missions have various operating conditions and the failure rate evolves with the latter. So, the reliability of the system depends also on the working conditions but with minimal maintenance activities, the reliability must be continuous over time. In Schutz et al. [10, a functional age was defined to ensure a continuous reliability. To illustrate this functional age, let's consider two consecutive missions $i$ and $j$. The reliability at the end of the mission $i$ (at time $\nu_{i}+\delta_{i}$ ) under condition $z_{i}$ must be equal to the reliability of the functional age (denoted $\nu_{j}$ ) under condition $z_{i}$. The functional age is given by:

$$
\nu_{m}=R^{-1}\left(\left[R\left(\nu_{m-1}+\delta_{m-1}\right)\right]^{\frac{z_{m}}{z_{m-1}}}\right)
$$

where $R(\cdot)$ and $R^{-1}(\cdot)$ respectively denote the reliability function and its inverse. Therefore, from the equation (1), the average number of failures can be expressed by:

$$
\phi(\mathcal{M})=\sum_{m=1}^{\operatorname{dim}(\mathcal{M})}\left(z_{m} \cdot \int_{\nu_{m}}^{\nu_{m}+\delta_{m}} \lambda(t) d t\right)
$$




\subsection{Systematic Preventive Maintenance Policy}

The first improved maintenance policy consists in planning preventive maintenance activities systematically after the completion of each mission. More precisely, the same preventive maintenance actions must be achieved after the $(\operatorname{dim}(\mathcal{M})-1)$ first missions. Indeed, after the last mission, a perfect preventive maintenance action is carried out. For this policy, total maintenance costs are specified by the following equation:

$$
\Gamma(\mathcal{M}, \rho)=C_{C M} \cdot \phi(\mathcal{M}, \rho)+C_{P M}(\rho) \cdot(\operatorname{dim}(\mathcal{M})-1)
$$

where the average number of failures is given by:

$$
\phi(\mathcal{M}, \rho)=\sum_{m=1}^{\operatorname{dim}(\mathcal{M})}\left(z_{m} \cdot \int_{\nu_{m, \rho}}^{\nu_{m, \rho}+\delta_{m}} \lambda(t) d t\right)
$$

From the equation (5), the average number of failures may seem identical to the equation (3) (minimal maintenance policy). However, the difference is in functional age expression. As preventive maintenance (system age reduction according to the effectiveness factor $\rho$ ) are carried out after the missions, functional age is expressed by:

$$
\nu_{m, \rho}= \begin{cases}(1-\rho) \cdot R^{-1}\left(\left[R\left(\nu_{m-1, \rho}+\delta_{m-1}\right)\right]^{\frac{z_{m}}{z_{m-1}}}\right) & \text { if } m>1 \\ 0 & \text { else }\end{cases}
$$

\section{Determination of the Optimal Effectiveness Factor}

The effectiveness factor "plays" a role in the quality of preventive action implementation. Due to this factor, preventive maintenance is considered imperfect and the system is returned to a state between ABAO (As Bad As Old) and AGAN (As Good As New). Therefore, it seems logical that this factor may also be involved in modeling the costs and lengths of preventive maintenance. For example, the competence of the operators can influence the quality of maintenance activities, but the maintenance action cost also depends on these skills.

Although costs may depend on the effectiveness factor, they are not necessarily proportional to the latter. Consequently, the cost of preventive action is based on a fixed one (e.g. parts and products used) and a variable one (qualification level, experience, etc.). Similarly, the length of preventive maintenance is composed of a fixed and a variable duration based on $\rho$. In this research, the cost and duration are modeled by:

$$
\begin{aligned}
& C_{P M}(\rho)=C_{P M, F}+C_{P M, V}(\rho) \\
& \mu_{P M}(\rho)=\mu_{P M, F}+\mu_{P M, V}{ }^{(\rho)}
\end{aligned}
$$

where $C_{P M, F}$ and $C_{P M, V}$ correspond respectively to the part of fixed and variable costs. Similarly, the fixed and variable durations are $\mu_{P M, F}$ and $\mu_{P M, V}$. 
As mentioned in section 2, the total time allocated to preventive actions is a percentage (noted $\alpha$ in the equation (9) of cumulative duration of all missions. For this systematic maintenance policy, the effectiveness factor is the same for all preventive actions. Thus, the maximum value of $r h o$ is obtained from equation (8) and its interval is given by:

$$
\rho \in] 0, \underbrace{\min \left(1, \frac{\log \left(-\frac{C_{P M, F} \cdot(\operatorname{dim}(\mathcal{M})-1)-\alpha \cdot \sum_{m=1}^{\operatorname{dim}(\mathcal{M})} \delta_{m}}{(\operatorname{dim}(\mathcal{M})-1)}\right)}{\log \left(C_{P M, V}\right)}\right)}_{\text {maximum value of } \rho}]
$$

Integral limits of the function $\phi_{\text {sys }}(\mathcal{M}, \rho)$ are too complex to analytically determine the $\rho$ factor. The latter will be determined by a numerical resolution.

This maintenance policy, compared to the minimal maintenance policy, can be further improved by determining the optimal value of the effectiveness factor for each preventive action.

\section{3 "Sporadic" Preventive Maintenance Policy}

This preventive maintenance policy is called "sporadic" because after each mission, preventive actions can be (or not) performed with diverse effectiveness factors. Basically, this model expression is very similar to the previous model. The total maintenance cost is given by:

$$
\Gamma(\mathcal{M}, P)=C_{C M} \cdot \phi(\mathcal{M}, P)+\sum_{m=1}^{\operatorname{dim}(\mathcal{M})-1} C_{P M}\left(\rho_{m}\right) \cdot\left\lceil\rho_{m}\right\rceil
$$

with $P$ the effectiveness factors vector of the first $(\operatorname{dim}(\mathcal{M})-1)$ missions and $\left\lceil\rho_{m}\right\rceil$ represents the ceil of the factor $\rho_{m}$. So, if the effectiveness factor is equal to 0 , no preventive maintenance is performed (one of type ABAO has no interest).

The average number of failures, for this policy, is expressed by:

$$
\phi(\mathcal{M}, P)=\sum_{m=1}^{\operatorname{dim}(\mathcal{M})}\left(z_{m} \cdot \int_{\nu_{m, \rho_{m}}}^{\nu_{m, \rho_{m}}+\delta_{m}} \lambda(t) d t\right)
$$

and the expression of the functional age remains unchanged (cf. equation (16)

This model resolution is more difficult. Indeed, there is not one decision variable but... $\operatorname{dim}(P)$ decision variables. Here again, the determination of these effectiveness factors cannot be computed analytically. Given the duration 
allocated to preventive maintenance actions, effectiveness factors must satisfy the following relationship:

$$
\sum_{m=1}^{\operatorname{dim}(P)} \mu_{P M}\left(\rho_{m}\right) \leq \alpha \cdot \sum_{m=1}^{\operatorname{dim}(\mathcal{M})} \delta_{m}
$$

Unlike the systematic maintenance policy, the $\rho$ factor can take any value in the interval $] 0,1[$.

The next section presents a numerical example where the objective consists in determining the effectiveness factor of preventive maintenance in the case of systematic and sporadic policies.

\section{Numerical Example}

Let's consider the following arbitrarily chosen input data to illustrate our model:

$-\lambda(t)=\frac{2.5}{300} \cdot\left(\frac{t}{300}\right)^{(2.5-1)}$ (Weibull distribution, shape $=2.5$, scale $=300$ )

$-C_{C M}=500 \mathrm{mu}$ (money unit)

$-C_{P M}=300+(600)^{\rho} \mathrm{mu}$

- $\mu_{P M}=2+(8)^{\rho}$ tu (time unit)

$-\alpha=0.05$

Various missions are presented in Table 1 .

Table 1. Missions to perform during the lease period

\begin{tabular}{|c||c|c|c|c|c|c|}
\hline Mission & 1 & 2 & 3 & 4 & 5 & 6 \\
\hline$\delta_{m}$ & 106 & 107 & 82 & 104 & 108 & 121 \\
$g_{m}$ & 1.24 & 1.48 & 1.41 & 1.42 & 1.28 & 1.12 \\
\hline
\end{tabular}

When maintenance actions performed are only of minimal type, the average number of failures is about 6.66 and the total cost of maintenance activities is $3331.5 \mathrm{mu}$. With this cost, it will be possible to estimate profit from the two preventive policies described in the previous section.

\subsection{Systematic Maintenance Policy}

As the total duration of all missions is $628 \mathrm{tu}$, the duration for preventive maintenance actions is $31,40 \mathrm{tu}(\alpha \cdot 628)$. After each mission, preventive activities can last, at the maximum, $6.28 \mathrm{tu}\left(\frac{\alpha \cdot 628}{6-1}\right)$. During this period allocated to (imperfect) preventive maintenance, the maximum value of effectiveness factor is 0.69. Based on maintenance costs given above, the total cost of maintenance activities amounted to $2289,7 \mathrm{mu}$ with $\rho=0.58$ and an average number of failures about 1.17. This first improved policy generates a profit of $31 \%$. When the effectiveness factor is less than 0.19 , this maintenance policy is less interesting than the minimal policy. This is due to the slight decrease in the number of failures compared to preventive actions cost. 


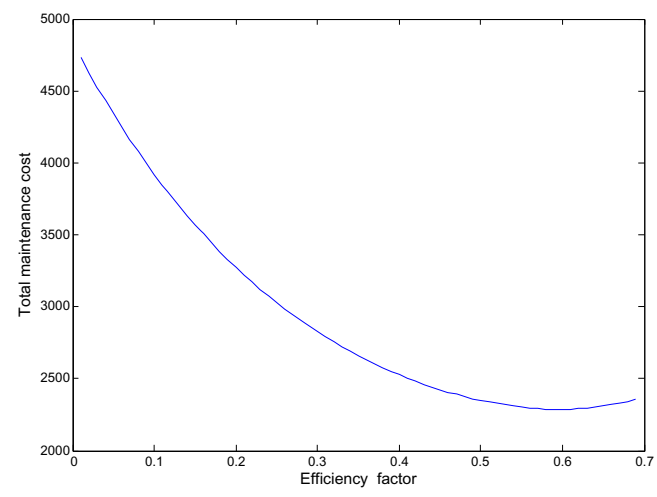

Fig. 1. Evolution of the total maintenance cost based on the effectiveness factor

\section{2 "Sporadic" Maintenance Policy}

As this preventive maintenance policy is more flexible, results are even better. From the numerical resolution, the best choice is to perform preventive maintenance activities after the second mission (with $\rho=0.74$ ) and after the fourth mission (with $\rho=0.78$ ). With these optimal decision variables, total maintenance cost is reduced by $46 \%$ compared to the minimal maintenance policy. The average number of failures is 1.87 , the cumulative duration of preventive maintenance actions is 13.72 . In this case, extra time $(31,4-13.72)$ can be used to add preventive maintenance actions to reduce the number of failures. However, total cost will be higher.

\section{Conclusion and Prospects}

In this paper, we considered a leased equipment for a defined horizon. During the lease period, the system was used in various working conditions. The latter affected the system, which degraded differently. To maintain a continuous reliability, functional age was introduced to generate equivalences between different operational conditions and working times. Thanks to this functional age, three maintenance policies have been studied. The first maintenance, minimal, is used to assess preventive maintenance policies benefit. Systematic preventive maintenance policy, characterized by the same preventive activities after each mission, give the best economical result. However, this result depends on the ratio between preventive and corrective costs (in the section referring to the numerical example, if $\rho<0.19$, this policy should be avoided). The "sporadic" policy allows preventive maintenance action after the chosen missions. It is therefore more flexible but the determination of the decision variables requires much more time. It would be interesting to use a meta-heuristic for solving this problem when the number of missions increases. 
Among possible perspectives, it would be interesting to study the case where the maximum duration after each preventive mission depends directly on mission duration. These maintenance policies may also be more realistic considering imperfect corrective actions and no negligible duration for these activities. The lessee can impose criteria such as equipment availability. In this case, preventive actions could be performed during missions. Another perspective would be to consider a set of different possible missions (with time windows). The aim would be to select missions to perform and to determine an optimal maintenance plan.

\section{References}

1. Barlow, R.E., Hunter, L.C.: Optimum Preventive maintenance policies. Operations Research 8(1), 90-100 (1960)

2. Cox, D.: Regression Models and Life-Tables. Journal of the Royal Statistical Society $34(2), 187-220(1972)$

3. Doyen, L., Gaudoin, O.: Classes of imperfect repair models based on reduction of failure intensity or virtual age. Reliability Engineering and System Safety 84(1), 45-56 (2004)

4. Jaturonnatee, J., Murthy, D.N.P., Boondiskulchok, R.: Optimal preventive maintenance of leased equipment with corrective minimal repairs. European Journal of Operational Research 174(1), 201-215 (2006)

5. Kijima, M., Morimura, H., Suzuki, Y.: Periodical replacement problem without assuming minimal repair. European Journal of Operational Research 37, 194-203 (1988)

6. Martorell, S., Sanchez, A., Vicente, S.: Age-dependent reliability model considering effects of maintenance and working conditions. Reliability Engineering and System Safety 64(1), 19-31 (1999)

7. Nakagawa, T.: Periodic and Sequential Preventive Maintenance Policies. Journal of Applied Probability 23(2), 536-542 (1986)

8. Nakagawa, T.: Sequential Imperfect Preventive Maintenance Policies. IEEE Transactions on Reliability 37(3), 295-298 (1988)

9. Pongpech, J., Murthy, D.N.P.: Optimal periodic preventive maintenance policy for leased equipment. Reliability Engineering \& System Safety 91(7), 772-777 (2006)

10. Schutz, J., Rezg, N.: An integrated strategy for efficient business plan and maintenance plan for systems with a dynamic failure distribution. Journal of Intelligent Manufacturing 24(21), 87-97 (2013)

11. Yeh, R.H., Chang, W.L.: Optimal threshold value of failure-rate for leased products with preventive maintenance actions. Mathematical and Computer Modelling 46(56), 730-737 (2007)

12. Yeh, R.H., Kao, K.C., Chang, W.L.: Optimal preventive maintenance policy for leased equipment using failure rate reduction. Computers \& Industrial Engineering 57(1), 304-309 (2009)

13. Yeh, R.H., Lo, H.C., Yu, R.Y.: A study of maintenance policies for second-hand products. Computers \& Industrial Engineering 60(3), 438-444 (2011)

14. Yeh, R.H., Kao, K.C., Chang, W.L.: Preventive-maintenance policy for leased products under various maintenance costs. Expert Systems with Applications 38(4), 3558-3562 (2011) 